Case Report

\title{
A Fatal Case of Concurrent Disseminated Tuberculosis, Pneumocystis Pneumonia, and Bacterial Septic Shock in a Patient with Rheumatoid Arthritis Receiving Methotrexate, Glucocorticoid, and Tocilizumab: An Autopsy Report
}

\author{
Shin-Ichiro Ohmura ${ }^{D},{ }^{1}$ Ryuhei Ishihara, ${ }^{1}$ Ayaka Mitsui, ${ }^{2}$ Yoshiro Otsuki, ${ }^{2}$ \\ and Toshiaki Miyamoto ${ }^{1}$ \\ ${ }^{1}$ Department of Rheumatology, Seirei Hamamatsu General Hospital, Shizuoka, Hamamatsu, Japan \\ ${ }^{2}$ Department of Pathology, Seirei Hamamatsu General Hospital, Shizuoka, Hamamatsu, Japan \\ Correspondence should be addressed to Shin-Ichiro Ohmura; s-omura0018@sis.seirei.or.jp
}

Received 17 May 2021; Accepted 30 August 2021; Published 7 September 2021

Academic Editor: Tsai Ching Hsu

Copyright (C) 2021 Shin-Ichiro Ohmura et al. This is an open access article distributed under the Creative Commons Attribution License, which permits unrestricted use, distribution, and reproduction in any medium, provided the original work is properly cited.

\begin{abstract}
Recently, treatment for rheumatoid arthritis has dramatically improved but increases the risk of bacterial and opportunistic infections. Herein, we report a fatal case of concurrent disseminated tuberculosis, pneumocystis pneumonia, and septic shock due to pyelonephritis caused by extended-spectrum $\beta$-lactamase-producing Escherichia coli in a patient with rheumatoid arthritis who received methotrexate, glucocorticoid, and tocilizumab. Despite undergoing intensive treatment, the patient developed respiratory failure and died after 7 days of admission. An autopsy indicated that pulmonary tuberculosis were the ultimate causes of death, while pyelonephritis was controlled.
\end{abstract}

\section{Introduction}

Rheumatoid arthritis (RA) is an autoimmune disease characterized by synovitis and structural damage to multiple joints. Treatment for RA has dramatically improved after the introduction of biologics. If patients do not show a good response during methotrexate (MTX) therapy, then rheumatologists administer biologics, including inhibitors of tumor necrosis factor $\alpha$, interleukin- (IL-) 6, and interactions between $\mathrm{T}$ cells and antigen-presenting cells by blocking cytotoxic $\mathrm{T}$ lymphocyte-associated protein 4 and Janus kinase in combination with MTX. However, these drugs increase the risk of bacterial and opportunistic infections, including tuberculosis (TB), cytomegalovirus infection, herpes zoster infection, and Pneumocystis jirovecii pneumonia (PCP) [1-4].

Patients with human immunodeficiency virus infection (HIV) with CD4 count below $200 / \mu \mathrm{L}$ experience opportunistic infections [5]. However, there are no surrogate markers to identify the development of opportunistic infections in non-HIV patients, including patients with RA.

Here, we report a case of a 79-year-old Japanese woman with RA who developed disseminated TB, PCP, and bacterial septic shock during MTX, glucocorticoid, and tocilizumab (TCZ) therapy.

\section{Case Presentation}

A 79-year-old woman with RA was referred to our hospital. She was diagnosed with RA because of polyarthritis and positive C-reactive protein (CRP) and anticyclic citrullinated peptide antibody. She had been receiving treatment with MTX and glucocorticoid for 1 year, although her disease activity could not be controlled with these drugs. Consequently, we initiated $40 \mathrm{mg}$ of adalimumab (ADA) for every 2 weeks. However, her disease activity did not improve 
TABLE 1: Laboratory tests before admission.

\begin{tabular}{|c|c|c|c|c|c|}
\hline Day after TCZ & $\mathrm{WBC}(/ \mu \mathrm{L})$ & Lymphocyte count $(/ \mu \mathrm{L})$ & $\mathrm{CRP}(\mathrm{mg} / \mathrm{dL})$ & Albumin $(\mathrm{g} / \mathrm{dL})$ & $\operatorname{IgG}(\mathrm{mg} / \mathrm{dL})$ \\
\hline Day 1 & 12820 & 1615 & 5.4 & 2.3 & 686 \\
\hline Day 15 & 8770 & 1666 & 0.09 & 2.7 & 524 \\
\hline Day 29 & 5570 & 1688 & 0.01 & 2.8 & 487 \\
\hline Day 44 & 4610 & 724 & 0.01 & 3.3 & N.A \\
\hline Day 69 (on admission) & 1600 & 221 & 0.59 & 3.0 & 294 \\
\hline
\end{tabular}

CRP, C reactive protein; IgG, immunoglobulin G; N.A, not available; TCZ, tocilizumab; WBC, leukocyte count.

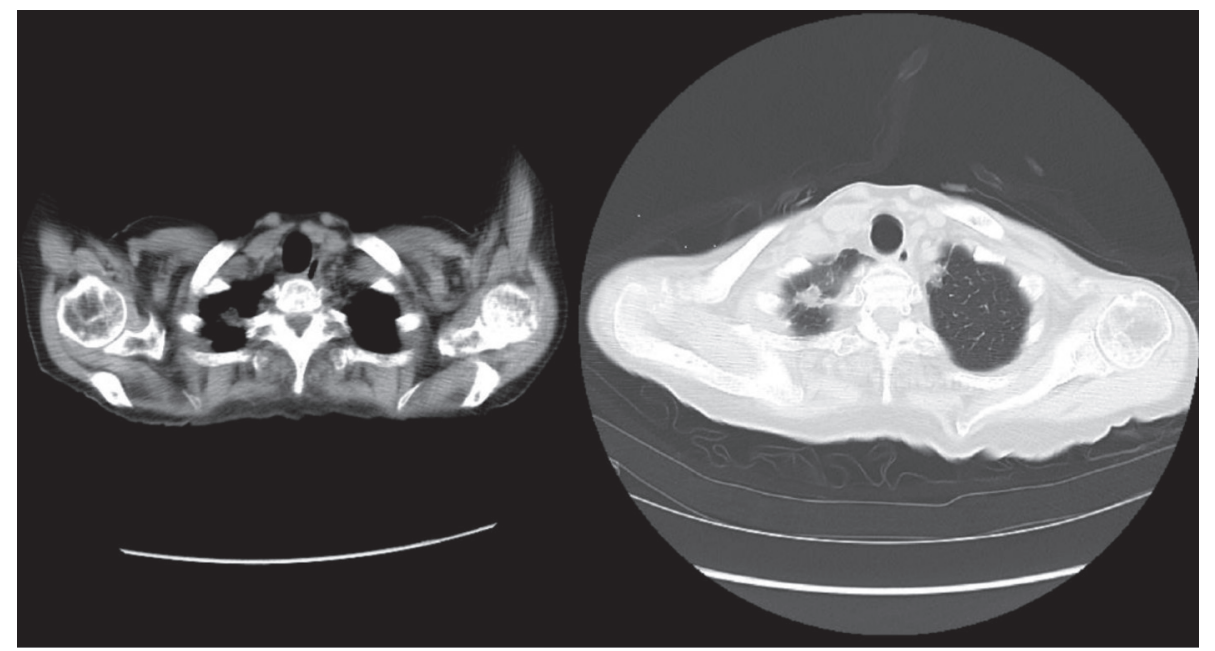

FIgURE 1: Chest CT showing thickening of the pleura and a nodule at the apex of the left lobe.

despite ADA treatment with 7 weeks, and $162 \mathrm{mg}$ of TCZ every two weeks was initiated in combination with $12 \mathrm{mg}$ of MTX every week and $12 \mathrm{mg}$ of methylprednisolone per day. Before the initiation of TCZ, she did not have a history of TB or come in contact with any patient with TB. Blood tests showed a leukocyte count of $12820 / \mu \mathrm{L}$, lymphocyte count of $1615 / \mu \mathrm{L}, \mathrm{CRP}$ of $5.4 \mathrm{mg} / \mathrm{dL}$, and immunoglobulin $\mathrm{G}$ (IgG) level of $686 \mathrm{mg} / \mathrm{dL}$ (Table 1). The T-SPOT-TB test is a type of $\mathrm{T}$ cells recognizing antigens specific to Mycobacterium tuberculosis, and serum $\beta$-D glucan was negative. She had no family history of TB. Chest computed tomography (CT) showed thickening of the pleura and a nodule at the apex of the left lobe (Figure 1). We did not administer anti-TB drugs because the T-SPOT-TB test and sputum test for TB were negative. Since we suspected her to have sulfamethoxazole/ trimethoprim (SMX/TMP) allergy, $300 \mathrm{mg}$ of inhaled pentamidine was administered every 4 weeks, as prophylaxis for PCP. However, her disease activity did not improve and TCZ was shortened every week. After every week TCZ treatment, her serum CRP level was $0.01 \mathrm{mg} / \mathrm{dL}$ within 1 month. However, the serum IgG levels gradually decreased, and lymphocyte count decreased on day 44 (Table 1). Two months after switching to TCZ, the patient was admitted to our hospital due to sudden high fever with a two-week history of generalized fatigue and cough. She was not in contact with anyone with an infection or coronavirus disease 2019, and she did not go to any infected areas. She was under treatment with $8 \mathrm{mg}$ of methylprednisolone per day, $12 \mathrm{mg}$ of MTX every week, and $162 \mathrm{mg}$ of TCZ every week for RA before admission.
On admission, her temperature was $39.1^{\circ} \mathrm{C}$, blood pressure was $70 / 53 \mathrm{mmHg}$, and respiration rate was 28 with a pulse rate of 136 beats per minute. Her blood oxygen saturation level using pulse oximetry was $96 \%$ under an oxygen flow of $10 \mathrm{~L} / \mathrm{min}$ via nonrebreathing oxygen mask with a reservoir bag. Her Glasgow Coma Scale score was 14: eye opening, 4; verbal response, 5; and motor response, 5. Her height was $149 \mathrm{~cm}$, and her body weight was $33.4 \mathrm{~kg}$. Her body mass index (BMI) was 15.0. Physical examination revealed mild and coarse crackles in the right and left sides of the chest. Blood tests showed a leukocyte count: $1600 / \mu \mathrm{L}$, neutrophil count: $1208 / \mu \mathrm{L}$, lymphocyte count: $221 / \mu \mathrm{L}$, hemoglobin: $10.7 \mathrm{~g} / \mathrm{dL}$, platelet count: $154000 / \mu \mathrm{L}$, total bilirubin: $1.5 \mathrm{mg} / \mathrm{dL}$, CRP: $0.59 \mathrm{mg} / \mathrm{L}$, lactate dehydrogenase: $509 \mathrm{U} / \mathrm{L}$, creatinine: $0.29 \mathrm{mg} / \mathrm{dL}$, prothrombin time: $12.5 \mathrm{sec}$, activated partial thromboplastin time: $26.5 \mathrm{sec}$, fibrinogen: $85 \mathrm{mg} / \mathrm{dL}$, D-dimer: $2.4 \mu \mathrm{g} / \mathrm{mL}$, Krebs von den Lungen-6 (KL-6): $423.3 \mathrm{U} / \mathrm{mL}$, pulmonary surfactant protein-D: $199 \mathrm{ng} / \mathrm{mL}$, and brain natriuretic peptide (BNP): $42.8 \mathrm{pg} / \mathrm{mL}$. The arterial blood gas analysis, while under an oxygen flow of $10 \mathrm{~L} / \mathrm{min}$ via nonrebreathing oxygen mask with a reservoir bag, showed $\mathrm{pH}$ : 7.504, partial pressure of arterial oxygen $\left(\mathrm{PaO}_{2}\right)$ : $159.0 \mathrm{mmHg}$, partial pressure of arterial carbon dioxide $\left(\mathrm{PaCO}_{2}\right): 33.4 \mathrm{mmHg}$, base excess: $3.2 \mathrm{mEq} /$ L, HCO3: $26.3 \mathrm{mEq} / \mathrm{L}$, and arterial oxygen saturation: $100 \%$. Calculated fraction of inspiratory oxygen $\left(\mathrm{FiO}_{2}\right)$ under an oxygen flow of $10 \mathrm{~L} / \mathrm{min}$ via nonrebreathing oxygen mask with a reservoir bag was 0.76 based on the previous report [6]. The sequential organ failure assessment score was 5. Cytomegalovirus pp65 antigenemia was negative. Serum 


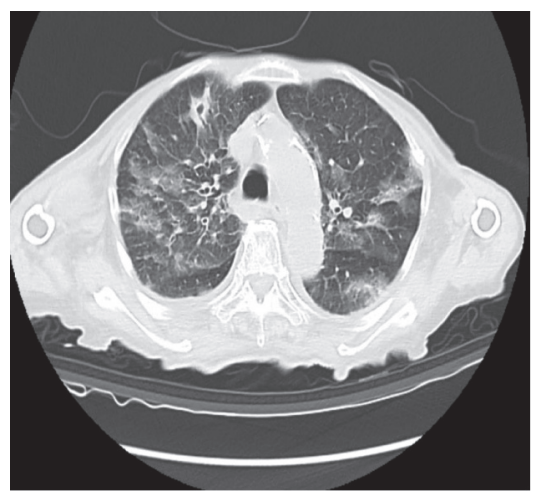

(a)

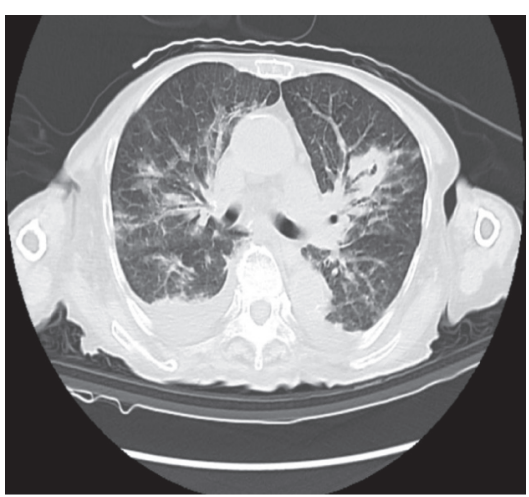

(b)

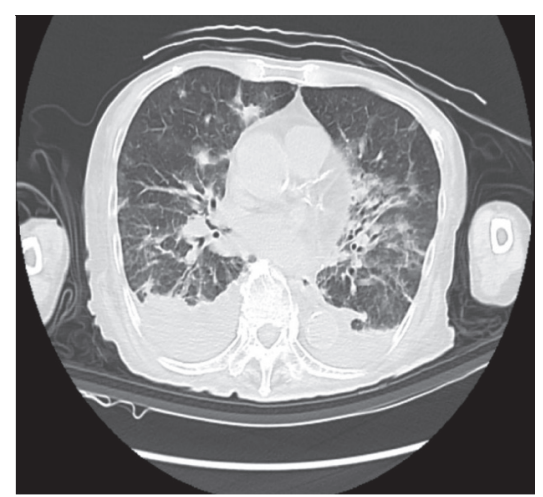

(c)

Figure 2: Chest CT image at the upper lobe level (a), at the carina level (b), and at the lower lobe level (c) on admission. Chest CT showed grand glass opacity in all fields with consolidation in the right and left lungs.

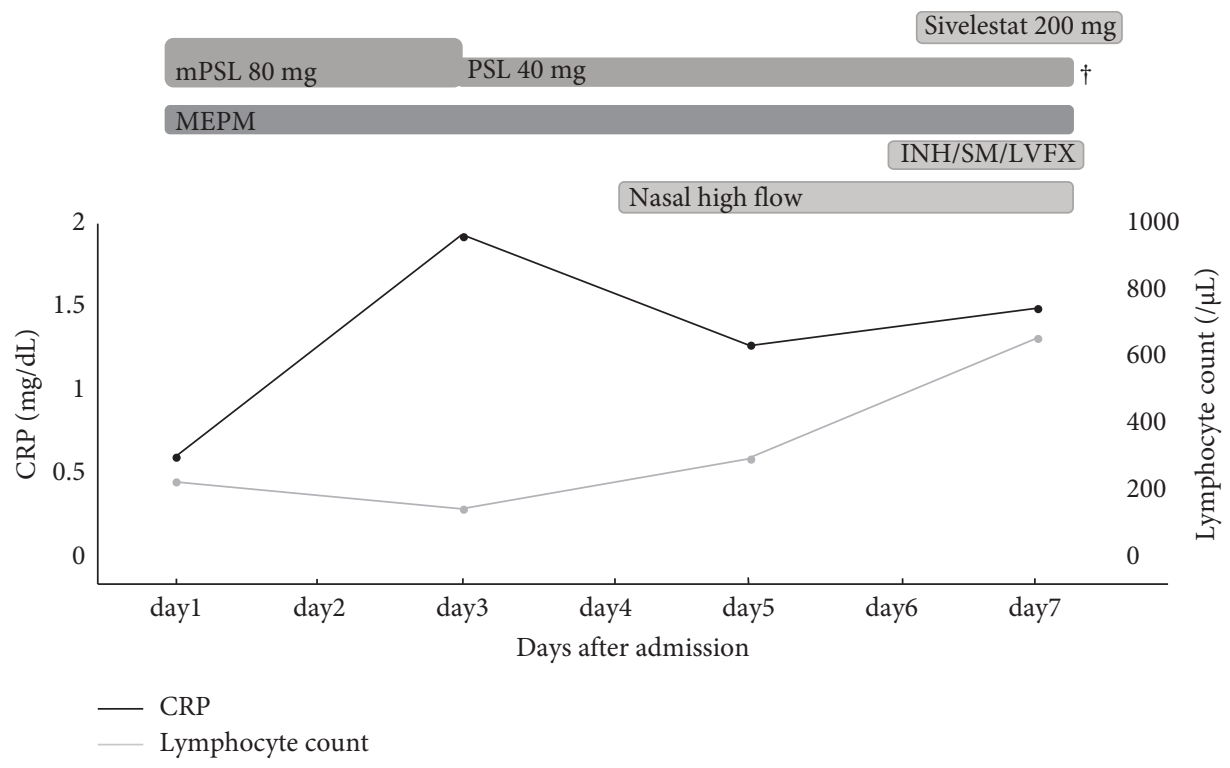

FIgURE 3: The clinical course. CRP, C-reactive protein; INH, isoniazid; LVFX, levofloxacin; MEPM; meropenem; mPSL, methylprednisolone; PSL, prednisolone; SM, streptomycin; TB, tuberculosis.

$\beta$-D glucan level was $286 \mathrm{pg} / \mathrm{mL}$, and the T-SPOT-TB test was positive. Antigen tests for the presence of species of Aspergillus, Candida, and Cryptococcus neoformans were all negative. The PCR-based sputum test for Pneumocystis jirovecii DNA was positive. The antigen test for coronavirus disease 2019 was negative. Streptococcus pneumoniae and Legionella pneumoniae urinary antigen tests were negative. CT scan showed grand glass opacity in all fields with consolidation within the right and left lungs (Figure 2) and mildly swollen kidneys without hydronephrosis. The echocardiography was normal. Her $\mathrm{PaO}_{2} / \mathrm{FiO}_{2}$ was $209.2 \mathrm{mmHg}$. She was diagnosed as having septic shock [7] and also diagnosed as acute respiratory distress syndrome (ARDS) based on the Berlin definition [8]. The clinical course is shown in Figure 3. She was treated with $3 \mathrm{~g}$ of meropenem per day. Because of worsening respiratory condition, the dose of methylprednisolone was increased to $80 \mathrm{mg}$ per day for her ARDS based on previous studies $[9,10]$. These treatments made her afebrile; however, the respiratory condition gradually worsened, and she required high-flow nasal oxygen on day 3. On the same day, the blood and urinary culture were positive for extended-spectrum $\beta$-lactamase-producing Escherichia coli. Since the result of the sputum test for TB was positive on day 6 , daily administration of antitubercular agents including, $200 \mathrm{mg}$ of isoniazid, $500 \mathrm{mg}$ of levofloxacin, and $500 \mathrm{mg}$ of streptomycin was initiated. However, the hypoxemia gradually worsened, and she died of respiratory failure on day 7 . With written permission from her family, an autopsy was performed. 


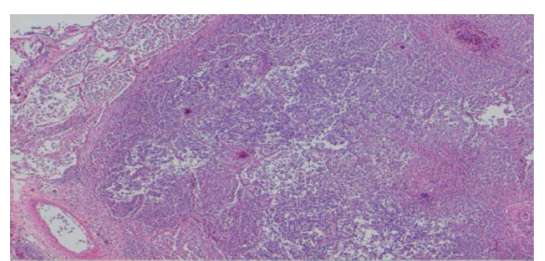

(a)

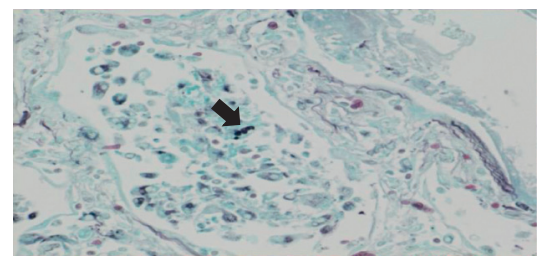

(d)

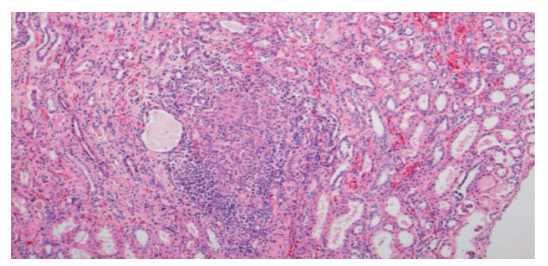

(g)

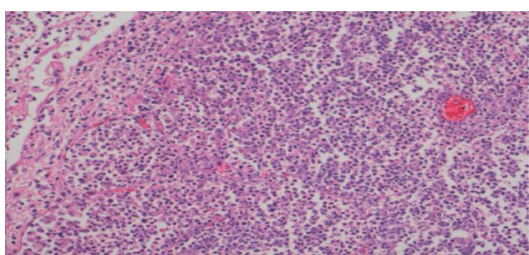

(b)

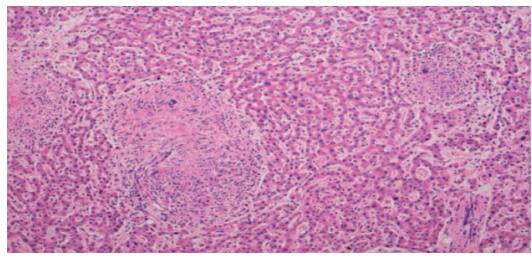

(e)

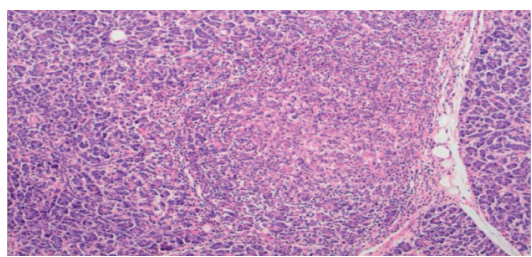

(h)

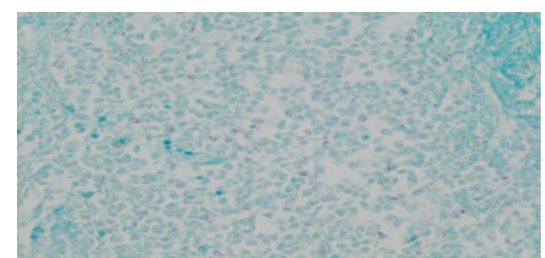

(c)

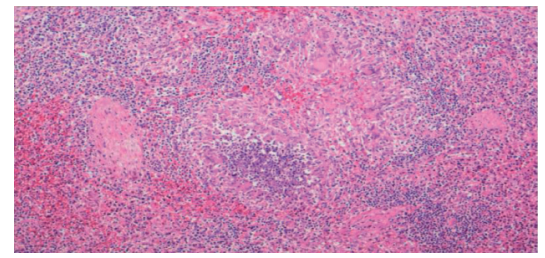

(f)

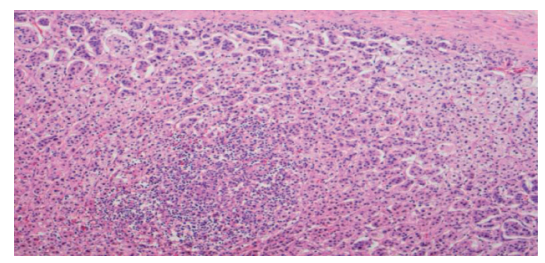

(i)

Figure 4: Excessive neutrophil accumulation with necrosis in the lung $((\mathrm{a}) \times 40$; (b) $\times 100)$. Numerous acid-fast bacilli with Ziehl-Neelsen stain $((\mathrm{c}) \times 400)$ and round to oval cysts with Grocott's methenamine silver nitrate stain $((\mathrm{d}) \times 400)$. Granulomas present in the liver (e), spleen (f), kidney (g), pancreas (h), and adrenal gland (i).

2.1. Autopsy (Figure 4). Bilateral lungs showed gross necrotic and cystic lesions of a maximum size of $30 \mathrm{~mm}$. Histologically, the necrotic lesions consisted infiltration of neutrophils, macrophages, and giant cells with severe inflammatory exudate. However, granulomatous formations were not observed. Ziehl-Neelsen staining aided the detection of acid-fast bacilli (Figure 4(a)-4(c)). These lesions seemed to be of primary TB. A few numbers of pneumocystis organisms were identified in the lungs using Grocott's methenamine silver nitrate staining (Figure 4(d)). Numerous granulomas were present as extrapulmonary lesions in the liver, spleen, kidneys, pancreas, and adrenal glands (Figures 4(e)-4(i)). Some of them contained caseous necrosis and/or Langhans giant cells. Mild lymphocyte infiltration was observed around the renal pelvis.

\section{Discussion}

We present a fatal case of RA with disseminated TB, PCP, and septic shock due to pyelonephritis caused by extendedspectrum $\beta$-lactamase-producing Escherichia coli treated with MTX, methylprednisolone, and TCZ.

To the best of our knowledge, there are no reports on patients with RA concurrently developing TB, PCP, and bacterial septic shock. In patients with HIV, Sheikholeslami et al. reported that $10 \%$ of the respiratory samples were coinfected with Mycobacterium tuberculosis and Pneumocystis jirovecii [11]. However, there is only one report on the development of coinfection with Mycobacterium tuberculosis and Pneumocystis jirovecii among patients with systemic rheumatic diseases [12]. Patients with RA develop infections more frequently than patients without RA do, and several reports have revealed that the use of biologicals increase infections, particularly pulmonary infections [13]. According to the mandatory postmarketing surveillance programs in Japan, reports have shown that $1.3 \%-2.2 \%$ of patients with RA treated with biologicals developed bacterial pneumonia, $0.18 \%-0.44 \%$ developed PCP, and $0.05 \%-0.28 \%$ developed TB [1-3]. In the case reported here, she did not receive prophylaxis for $\mathrm{TB}$ because she had no history of $\mathrm{TB}$, and her T-SPOT-TB test and sputum test for Mycobacterium tuberculosis were negative. However, several investigators reported that older age, low BMI, immunosuppressive treatment, lymphocytopenia, and hypoalbuminemia are factors for the false-negative T-SPOT-Tb assay $[14,15]$. In our patient, she was older age, had hypoalbuminemia, low BMI, and received immunosuppressive treatment, which might have suggested false-negative for the T-SPOT-Tb assay. According to the Japan College of Rheumatology, patients who are suspected to have latent $\mathrm{TB}$ include the history of TB, chest X-ray findings compatible with old TB, and positive interferon-gamma release assay or tuberculin test should receive prophylaxis if physicians administer biologics [16]. In our patient, chest CT before TCZ treatment showed thickening of the pleura and a nodule, suggesting that prophylaxis might have required for $\mathrm{TB}$ despite no history of TB, and the T-SPOT-TB test and sputum test for Mycobacterium tuberculosis were negative.

Alternatively, an age of at least 65 years, a daily dose of prednisolone of at least $6 \mathrm{mg}$, and the presence of coexisting 
pulmonary disease are the risk factors for PCP in patients with RA receiving infliximab; patients with two or three of these risk factors had a significantly higher cumulative probability of PCP than patients with no risk factors [17]. Our patient had two risk factors, including old age and glucocorticoid use, which showed a high risk for the development of PCP [17]. She received inhaled pentamidine every 4 weeks; however, she developed PCP. Schneider et al. reported that inhaled pentamidine once a month was less effective as primary prophylaxis against PCP than SMX/ TMP in patients with HIV [18].

Biologics and glucocorticoids increase the risk of hospital-acquired infections [4]. In particular, glucocorticoids are associated with a dose-dependent increase in the risk of serious infections [19]. Our patient received $8 \mathrm{mg} /$ day of methylprednisolone in combination with MTX and TCZ, which is consistent with previous studies [4, 19]. In addition, glucocorticoids decrease serum IgG levels, which is associated with severe bacterial infections [20,21]. According to the Clinical Guidelines for Immunoglobulin Use, patients with secondary antibody deficiency with a serum IgG level $<500 \mathrm{mg} / \mathrm{dL}$ should receive intravenous immunoglobulin therapy [22]. In our patient, the serum IgG level was $294 \mathrm{mg} /$ $\mathrm{dL}$ on admission and $487 \mathrm{mg} / \mathrm{dL}$ on day 29; this showed secondary antibody deficiency. In addition, our patient received ADA treatment before TCZ. Anti-TNF inhibitorsexperienced patients were also associated with significant serious infections and the incidence rate of TB after the initiation of TNF- $\alpha$ inhibitors was very high $[23,24]$. On the other hand, the risk of infection with TCZ was similar to that with other biologicals [25], and TCZ does not influence on the IFN- $\gamma$ synthesis by TB antigens [26]. However, TCZ masks clinical symptoms and decreases serum CRP levels [27], and early diagnosis of infection is very difficult in patients receiving TCZ.

Subesinghe et al. reported that serum lymphocyte nadir counts below $1000 / \mu \mathrm{L}$ in patients with RA, particularly below $500 / \mu \mathrm{L}$, were at high risk of serious infections; this reduction appears within the immediate 30 days prior to severe infections [28]. In our patient, the serum lymphocyte counts on day 29 after TCZ was normal, although it decreased on day 44. On admission, she had a history of generalized fatigue with cough for two weeks. The serum lymphocyte counts increased after admission, despite the high-dose glucocorticoid therapy. These results show that serum lymphocytopenia preceded infection, and serum lymphocyte count might be a useful marker to help predicting opportunistic infections as well as the other severe infections.

In conclusion, patients with RA may develop severe opportunistic infections, in particular receiving biologics. We must be aware of the potential of opportunistic infections and may consider prophylaxis for opportunistic infections in these patients with high risk.

\section{Consent}

A written informed consent for this case report has been obtained from patient's family.

\section{Conflicts of Interest}

The authors declare that they have no conflicts of interest.

\section{Authors' Contributions}

All authors approved the final version of this article. MA and OY reviewed the all autopsies. SO drafted the article, had full access to all of the data, and was responsible for the organization and coordination of the case.

\section{References}

[1] T. Takeuchi, Y. Tatsuki, Y. Nogami et al., "Postmarketing surveillance of the safety profile of infliximab in 5000 Japanese patients with rheumatoid arthritis," Annals of the Rheumatic Diseases, vol. 67, no. 2, pp. 189-194, 2008.

[2] T. Koike, M. Harigai, S. Inokuma et al., "Postmarketing surveillance of the safety and effectiveness of etanercept in Japan," Journal of Rheumatology, vol. 36, no. 5, pp. 898-906, 2009.

[3] T. Koike, M. Harigai, S. Inokuma et al., "Postmarketing surveillance of tocilizumab for rheumatoid arthritis in Japan: interim analysis of 3881 patients," Annals of the Rheumatic Diseases, vol. 70, no. 12, pp. 2148-2151, 2011.

[4] A. L. Smitten, H. K. Choi, M. C. Hochberg et al., "The risk of hospitalized infection in patients with rheumatoid arthritis," Journal of Rheumatology, vol. 35, no. 3, pp. 387-393, 2008.

[5] J. E. Kaplan, C. Benson, K. H. Holmes et al., "Guidelines for the prevention and treatment of opportunistic infections in HIV-infected adults and adolescents: recommendations from the centers for disease control and prevention, the national institutes of health, and the HIV medicine association of the infectious diseases society of America," Morbidity and Mortality Weekly Report, vol. 58, 2009.

[6] T. Yorozu, K. Moriyama, A. Motoyasu, M. Kotani, K. Uzawa, and T. Kohyama, "Effect of fit of non-rebreathing oxygen mask with a reservoir bag at various oxygen flow rates on inspiratory oxygen concentrations using a high-fidelity patient simulator," Journal of Japanese Society of Intensive Care Medicine, vol. 21, pp. 607-617, 2014.

[7] M. Singer, C. S. Deutschman, C. W. Seymour et al., "The third international consensus definitions for sepsis and septic shock (sepsis-3)," Journal of the American Medical Association, vol. 315, no. 8, pp. 801-810, 2016.

[8] ARDS Definition Task Force, V. M. Ranieri, V. M. Ranieri et al., "Acute respiratory distress syndrome: the berlin definition," Journal of the American Medical Association, vol. 307, no. 23, pp. 2526-2533, 2012.

[9] G. U. Meduri, E. Golden, A. X. Freire et al., "Methylprednisolone infusion in early severe ARDS," Chest, vol. 131, no. 4, pp. 954-963, 2007.

[10] N. Seam, G. U. Meduri, H. Wang et al., "Effects of methylprednisolone infusion on markers of inflammation, coagulation, and angiogenesis in early acute respiratory distress syndrome," Critical Care Medicine, vol. 40, no. 2, pp. 495-501, 2012.

[11] M. F. Sheikholeslami, J. Sadraei, P. Farnia, M. Forozandeh Moghadam, and H. Emadi Kochak, "Co-infection of mycobacterium tuberculosis and pneumocystis jirovecii in the Iranian patients with human immunodeficiency virus," Jundishapur Journal of Microbiology, vol. 8, no. 2, Article ID e17254, 2015. 
[12] A. K. Janmeja, P. R. Mohapatra, M. R. Shivaprakash, A. Khurana, and D. Aggarwal, "Concurrent infection of pneumocystis pneumonia and pulmonary tuberculosis in an HIV-seronegative patient," Indian Journal of Chest Diseases and Allied Sciences, vol. 50, no. 4, pp. 369-371, 2008.

[13] M. F. Doran, C. S. Crowson, G. R. Pond, W. M. O'Fallon, and S. E. Gabriel, "Predictors of infection in rheumatoid arthritis," Arthritis \& Rheumatism, vol. 46, no. 9, pp. 2294-2300, 2002.

[14] L. Pan, H. Jia, F. Liu et al., "Risk factors for false-negative T-SPOT.TB assay results in patients with pulmonary and extra-pulmonary TB," Journal of Infection, vol. 70, no. 4, pp. 367-380, 2015.

[15] Y. Kobashi, T. Sugiu, H. Shimizu et al., "Clinical evaluation of the T-SPOT.TB test for patients with indeterminate results on the QuantiFERON TB-2G test," Internal Medicine, vol. 48, no. 3, pp. 137-142, 2009.

[16] H. Tokuda, M. Harigai, H. Kameda et al., "Consensus statements for medical practice: biological agents and lung disease [abridged english translation by the Japanese respiratory society]," Respiratory Investigation, vol. 55, no. 3, pp. 229-251, 2017.

[17] M. Harigai, R. Koike, and N. Miyasaka, "Pneumocystis pneumonia associated with infliximab in Japan," New England Journal of Medicine, vol. 357, no. 18, pp. 1874-1876, 2007.

[18] M. M. E. Schneider, A. I. M. Hoepelman, J. K. M. E. Schattenkerk et al., "A controlled trial of aerosolized pentamidine or trimethoprim-sulfamethoxazole as primary prophylaxis against pneumocystis carinii pneumonia in patients with human immunodeficiency virus infection," New England Journal of Medicine, vol. 327, no. 26, pp. 1836-1841, 1992.

[19] M. D. George, J. F. Baker, K. Winthrop et al., "Risk for serious infection with low-dose glucocorticoids in patients with rheumatoid arthritis: a cohort study," Annals of Internal Medicine, vol. 173, no. 11, pp. 870-878, 2020.

[20] H. D. Ochs and C. I. Edvard Smith, "X-linked agammaglobulinemia a clinical and molecular analysis," Medicine, vol. 75, no. 6, pp. 287-299, 1996.

[21] S. Shah, K. Jaggi, K. Greenberg, and D. Geetha, "Immunoglobulin levels and infection risk with rituximab induction for anti-neutrophil cytoplasmic antibody-associated vasculitis," Clinical Kidney Journal, vol. 10, no. 4, pp. 470-474, 2017.

[22] Clinical Guidelines for Immunoglobulin Use, 2nd edition, 2011.

[23] J. A. Singh, C. Cameron, S. Noorbaloochi et al., "Risk of serious infection in biological treatment of patients with rheumatoid arthritis: a systematic review and meta-analysis," The Lancet, vol. 386, no. 9990, pp. 258-265, 2015.

[24] F. Wolfe, L. Caplan, and K. Michaud, "Treatment for rheumatoid arthritis and the risk of hospitalization for pneumonia: associations with prednisone, disease-modifying antirheumatic drugs, and anti-tumor necrosis factor therapy," Arthritis \& Rheumatism, vol. 54, no. 2, pp. 628-634, 2006.

[25] H. Yun, F. Xie, E. Delzell et al., "Comparative risk of hospitalized infection associated with biologic agents in rheumatoid arthritis patients enrolled in medicare," Arthritis \& Rheumatology, vol. 68, no. 1, pp. 56-66, 2016.

[26] A. Ogata, M. Mori, S. Hashimoto et al., "Minimal influence of tocilizumab on IFN- $\gamma$ synthesis by tuberculosis antigens," Modern Rheumatology, vol. 20, no. 2, pp. 130-133, 2010.

[27] H. Fujiwara, N. Nishimoto, Y. Hamano et al., "Masked early symptoms of pneumonia in patients with rheumatoid arthritis during tocilizumab treatment: a report of two cases," Modern Rheumatology, vol. 19, no. 1, pp. 64-68, 2009.
[28] S. Subesinghe, A. Kleymann, A. I. Rutherford, K. Bechman, S. Norton, and J. Benjamin Galloway, "The association between lymphopenia and serious infection risk in rheumatoid arthritis," Rheumatology, vol. 59, no. 4, pp. 762-766, 2020. 